\title{
Sensitivity of ecosystem parameters to simulated satellite ocean color data using a coupled physical-biological model of the North Atlantic
}

\author{
by Jim Gunson $^{1,2}$, Andreas Oschlies ${ }^{3}$ and Véronique Garçon ${ }^{1}$
}

\begin{abstract}
A means of assimilating simulated satellite ocean color data with a coupled physical-biological model of the North Atlantic Ocean is implemented, allowing the relative sensitivities of different biological parameters to those data to be investigated. The model consists of an eddy-permitting general circulation model derived from the WOCE Community Modeling Effort and a nitrogenbased, four-compartment NPZD marine ecosystem model. Many of the parameters in marine ecosystem models are poorly known and via assimilation, we hope to better constrain their values. The control parameters chosen for the variational assimilation are the model parameters involved in parameterizations of recycling as these are the most poorly known. Simulated observations are taken while following several floats seeded in varying dynamical biogeochemical provinces of the North Atlantic model domain over a six-month period. Twin experimental results show that, for the given functional forms of growth, mortality and grazing, the following parameters can be successfully recovered from simulated satellite ocean color data: nitrate and detrital recycling parameters in the trade wind domain, zooplankton parameters at higher latitudes (westerly wind and polar domains), and the phytoplankton mortality rate in all regions. By simultaneously assimilating ocean color data in different biological provinces, it becomes possible to successfully constrain all ecosystem parameters at once.
\end{abstract}

\section{Introduction}

In order to study the processes by which carbon passes from the atmosphere down to the deep ocean, biogeochemical models have been coupled with realistic physical models of the oceanic circulation (Sarmiento et al., 1993; Oschlies and Garçon, 1998, 1999). One of the main problems is determining the biological model parameters for coupled models at the basin-scale. With the ocean color data sets that are now available from the POLDER, OCTS and SeaWiFS sensors it will be possible to use these data as a measure of surface chlorophyll concentration, to constrain the modeled biological processes. The parameter

1. UMR5566/LEGOS, Centre National de la Recherche Scientifique, 18 Avenue Edouard Belin, 31401 Toulouse Cedex 4, France.

2. Present address: The UK Meteorological Office, London Road, Bracknell, Berks RG12 2SZ, United Kingdom.email:jgunson@meto.gov.uk

3. Institut für Meereskunde an der Universität Kiel, Düsternbrooker Weg 20, 24105 Kiel, Germany. 
values, and eventually the parameterizations, may thus be improved, and a better estimate can be made of primary and export production in the ocean.

The physical ocean models in use can provide descriptions of the ocean circulation that are adequate for studies of the carbon cycle (Oschlies and Garçon, 1999). The ecosystem models that may be coupled to the physical models to represent plankton and nutrient dynamics are, of computational necessity, rather simplified. As part of JGOFS, Evans and Garçon (1997) compiled and compared a representative selection of biogeochemical models that are in use. An aim of JGOFS is to successfully model the observed spatial and temporal distributions of primary production, phytoplankton, chlorophyll, $\mathrm{pCO}_{2}$ and $\mathrm{TCO}_{2}$. At issue is whether the observed variability can be captured in a basin-scale coupled model. The North Atlantic basin covers many different dynamical biogeochemical provinces (Platt et al., 1995) within which different factors affecting plankton dynamics will be dominant. The regional variation of the biological model parameters in a basin-scale coupled model needs to be investigated.

Many of the parameters of plankton dynamics that are used in coupled models are poorly known. Previous studies have focused on assimilating ecosystem models with in situ observations in order to make better estimates of model parameters. Fasham and Evans (1995) fit a seven-compartment nitrogen-based ecosystem model (Fasham et al., 1990) to data from the North Atlantic Bloom Experiment. Matear (1995) fit the same ecosystem model to data from Station Papa in the North Pacific. A model descended from the Fasham et al. (1990) model was also used by Hurtt and Armstrong (1996) to fit data from the Bermuda Atlantic Time Series Study. In these studies rather rudimentary ocean physics were used. Horizontal advection was ignored and a homogeneous mixed layer with a flux boundary condition at the base was implemented. Prunet et al. (1996) fit a one-dimensional coupled physical/biogeochemical model (four-compartments) to Station Papa data. Although the ecosystem models used in these studies are all very similar, many of the parameterizations employed by the different authors are quite different. Also the temporal coverage among the studies varied: Matear (1995) and Hurtt and Armstrong (1996) were able to use averages of several years of data; Fasham and Evans (1995) used a single year of data as if it were steadily repeating; and Prunet et al. (1996) used two years of observations. Moreover, the studies ranged over different biogeochemical provinces where quite different factors influence the plankton dynamics.

Recently, Evans (1999) repeated the study of Fasham and Evans (1995) and assessed how these studies are addressing the goals of JGOFS. An objective of all of these studies was to test different parameterizations of biogeochemical processes, which involved estimating parameter values. In particular, attention was focused on the source and sink terms affecting phytoplankton growth and also on the relative importance of different in situ data types and sampling frequencies. However, it is difficult to make intercomparisons of the parameter estimations performed in these studies, as the models all used very different parameterizations of biogeochemical processes (see Evans and Garçon (1997) for a full review of the parameterizations). Also a reason for the diversity of model configurations and parameterizations is that the models were designed to study processes in different 
biogeographical provinces. In the present study we propose a unifying approach that starts from the hypothesis that a single model and a unique parameter set can describe the observed chlorophyll concentration in all provinces of the open North Atlantic Ocean.

In all of the previous studies a quadratic cost function is defined that expresses the misfit between the model and the data. One proceeds to minimize the cost function arguing that at the minimum of the cost function, one has found the model parameters most likely to produce the given data.

Because ecosystem dynamics are nonlinear, finding the minimum of the cost function is not straightforward. Most studies (Fasham et al., 1990; Prunet et al., 1996), starting from some $a$ priori values for the model parameters, calculate the gradient of the cost function with respect to the model parameters. This then allows a search for the minimum nearest to the a priori values of the model parameters. A disadvantage of this approach is that when the model dynamics are nonlinear the minimization may stop at some local minimum and not at the global minimum of the cost function. The minimization may also be performed by simulated annealing (Matear, 1995; Hurtt and Armstrong, 1996) whereby thousands of random steps are taken in search of the minimum. Using this approach the global minimum may be found but it is computationally expensive.

Here we will use variational data assimilation, the adjoint method, to fit a fourcompartment nitrogen-based model to simulated ocean color data. This method was employed previously by Lawson et al. (1996), who also used simulated data with a five-compartment model and zero-dimensional physics. Simulated data allow twin experiments to be performed, for which the global minimum is known in advance, and one minimizes the cost function in order to assess the sensitivity of the model to the data. In practice, using real data with a nonlinear model, there may not be a global minimum. There may be many minima of the cost function whose values are acceptably small, that is, the model agrees with the data to within the observational error. In such situations, the choice of a priori values for the model parameters will determine the minimum of the cost function.

We attempt to provide a more comprehensive physical setting for the assimilation by using the physical circulation taken from a 3D eddy-permitting general circulation model of the North Atlantic Ocean (Oschlies and Garçon, 1999). A one-dimensional biological model is implemented that follows the flow field produced by the ocean model. Hence the effects of horizontal advection are taken into account, and there is assumed to be no horizontal mixing. Ten floats are seeded in different locations around the North Atlantic model domain and followed over six months from November through April. An adjoint method is used to calculate the sensitivity of modeled ocean color to variations in the biological model parameters. This is done regionally and globally over the North Atlantic model domain.

In the next section the physical and biogeochemical components of the coupled model are described. The strategy of assimilation is then presented in Section 3. In Section 4, some experimental results are presented that illustrate the different sensitivities of simulated surface chlorophyll to the various biological model parameters in different parts of the ocean. Examining the gradient information given by the adjoint model can provide 
some insight into how the ecosystem model parameters depend upon the data. Finally Section 5 contains a discussion and some conclusions.

\section{Coupled physical-biological modeling}

\section{a. Physical component}

The ocean model used here is based on the Geophysical Fluid Dynamics Laboratory (GFDL) Modular Ocean Model (MOM, version 1.1), a primitive-equation ocean circulation model. In the configuration set up for the Community Modeling Effort (CME) by Bryan and Holland (1989), it covers the Atlantic Ocean from $15 \mathrm{~S}$ to $65 \mathrm{~N}$ with a meridional grid spacing of $1 / 3^{\circ}$ and a zonal grid spacing of $2 / 5^{\circ}$. There are 37 levels in the vertical with eight in the top 100 meters (see Table 1). A turbulent kinetic energy mixed-layer scheme (Gaspar et al., 1990) is implemented which allows vertical structure in the biological variables to develop within the mixed layer.

The model is forced with climatological data sets: monthly mean wind stress from Hellerman and Rosenstein (1983), monthly mean surface salinity from Levitus (1982), and monthly mean heat fluxes from Han (1984) and Barnier et al. (1995). More details on the configuration of the physical model may be found in Oschlies and Garçon (1999).

\section{b. Biological component}

Out of the large range of marine ecosystem models that could be used (Evans and Garçon, 1997), we have chosen one that may readily be coupled with a basin-scale eddy-permitting circulation model. The ecosystem dynamics are represented by a nitrogenbased $N-P-Z-D$ (nitrate, phytoplankton, zooplankton, detritus) pelagic model. The evolution of any biological tracer concentration $C_{i}$ is determined by an advective-diffusive equation that is integrated at every grid point of the physical model,

$$
\frac{\partial C_{i}}{\partial t}=-\nabla \cdot \mathbf{u} C_{i}-A_{\rho} \nabla^{4} C_{i}+\frac{\partial}{\partial z}\left(K_{\rho} \frac{\partial C_{i}}{\partial z}\right)+\operatorname{sms}\left(C_{i}\right)
$$

where the terms on the right-hand side represent: three-dimensional advection of the tracer by the velocity field, biharmonic horizontal diffusion, and vertical mixing with turbulent diffusion coefficient $K_{\rho}$. The last term on the right-hand side is the source-minus-sink term for each of the biological tracers, given by

$$
\begin{aligned}
& \operatorname{sms}(P)=J(z, t, N) P-G(P) Z-\mu_{P} P \\
& \operatorname{sms}(Z)=\gamma_{1} G(P) Z-\gamma_{2} Z-\mu_{Z} Z^{2} \\
& \operatorname{sms}(D)=\left(1-\gamma_{1}\right) G(P) Z+\mu_{P} P+\mu_{Z} Z^{2}-\mu_{D} D-w_{s} \frac{\partial D}{\partial z} \\
& \operatorname{sms}(N)=\mu_{D} D+\gamma_{2} Z-J(z, t, N) P
\end{aligned}
$$

where the meaning of each term is given in the list of model parameters in Table 2. 
Table 1. Level depths.

\begin{tabular}{|c|c|c|c|}
\hline $\begin{array}{l}\text { Model } \\
\text { level }\end{array}$ & $\begin{array}{l}\text { Depth of } \\
\text { grid point }\end{array}$ & $\begin{array}{l}\text { Depth of grid } \\
\text { box bottom }\end{array}$ & $\begin{array}{c}\text { Thickness o } \\
\text { grid box }\end{array}$ \\
\hline 1 & 5.50 & 11.00 & 11.00 \\
\hline 2 & 17.00 & 23.00 & 12.00 \\
\hline 3 & 29.00 & 35.00 & 12.00 \\
\hline 4 & 41.00 & 47.00 & 12.00 \\
\hline 5 & 53.00 & 59.00 & 12.00 \\
\hline 6 & 65.50 & 72.00 & 13.00 \\
\hline 7 & 78.50 & 85.00 & 13.00 \\
\hline 8 & 91.50 & 98.00 & 13.00 \\
\hline 9 & 104.50 & 111.00 & 13.00 \\
\hline 10 & 118.50 & 126.00 & 15.00 \\
\hline 11 & 140.50 & 155.00 & 29.00 \\
\hline 12 & 179.55 & 204.09 & 49.09 \\
\hline 13 & 232.60 & 261.10 & 57.01 \\
\hline 14 & 295.03 & 328.95 & 67.85 \\
\hline 15 & 370.21 & 411.47 & 82.52 \\
\hline 16 & 462.51 & 513.54 & 102.07 \\
\hline 17 & 577.37 & 641.19 & 127.65 \\
\hline 18 & 721.47 & 801.74 & 160.55 \\
\hline 19 & 900.89 & 1000.04 & 198.30 \\
\hline 20 & 1125.04 & 1250.04 & 250.00 \\
\hline 21 & 1375.04 & 1500.04 & 250.00 \\
\hline 22 & 1625.04 & 1750.04 & 250.00 \\
\hline 23 & 1875.04 & 2000.04 & 250.00 \\
\hline 24 & 2125.04 & 2250.04 & 250.00 \\
\hline 25 & 2375.04 & 2500.04 & 250.00 \\
\hline 26 & 2625.04 & 2750.04 & 250.00 \\
\hline 27 & 2875.04 & 3000.04 & 250.00 \\
\hline 28 & 3125.04 & 3250.04 & 250.00 \\
\hline 29 & 3375.04 & 3500.04 & 250.00 \\
\hline 30 & 3625.04 & 3750.04 & 250.00 \\
\hline 31 & 3875.04 & 4000.04 & 250.00 \\
\hline 32 & 4125.04 & 4250.04 & 250.00 \\
\hline 33 & 4375.04 & 4500.04 & 250.00 \\
\hline 34 & 4625.04 & 4750.04 & 250.00 \\
\hline 35 & 4875.04 & 5000.04 & 250.00 \\
\hline 36 & 5125.04 & 5250.04 & 250.00 \\
\hline 37 & 5375.04 & 5500.04 & 250.00 \\
\hline
\end{tabular}

The phytoplankton growth rate uses the minimum of light- and nutrient-limited growth, i.e.,

$$
J(z, t, N)=\min \left(J(z, t), J_{\max } \frac{N}{k_{1}+N}\right)
$$

where $J(z, t)$ denotes the purely light-limited growth rate, $J_{\max }$ is the light-saturated growth 
Table 2. Values of biological model parameters (EP85: Oschlies and Garçon, 1998b, after Evans and Parslow, 1985).

\section{Parameter}

Phytoplankton $(P)$ Coefficients

integration method for daily growth rate initial slope of P-I curve

photosynthetically active radiation

light attenuation due to water

light attenuation by phytoplankton

maximum growth rate parameters

half-saturation constant for $\mathrm{N}$ uptake

specific mortality rate

Zooplankton $(Z)$ Coefficients

assimilation efficiency

maximum grazing rate

prey capture rate

(quadratic) mortality

excretion
Symbol Value

EP85

$\alpha$

$P A R$

0.025

0.43

Detrital $(D)$ Coefficients

remineralization rate

sinking velocity $k_{w}$

$k_{c}$

$a$

$b$

$c$

$K_{1}$

$\mu_{P}$

$\gamma_{1}$

$g$

$\varepsilon$

$\mu_{Z}$

$\gamma_{2}$

0.04

0.03

0.6

1.066

1.0

0.5

0.03

0.75

2.0

1.0

0.20

0.03

$\begin{array}{lll}\mu_{D} & 0.05 & \mathrm{~d}^{-1} \\ w_{s} & 5.0 & \mathrm{~m} \mathrm{~d}^{-1}\end{array}$

rate and $k_{1}$ is the half-saturation constant for $N$ uptake by $P$. A detailed presentation of the calculation of $J(z, t)$, over the model domain as a function of time, follows Evans and Parslow (1985). The analytical integral of the growth rate includes a vertical integral over each grid box (see Oschlies and Garçon (1999) for more details). A consequence of lightvs. nutrient-limited growth is that at low latitudes phytoplankton respond to changes in nutrient supply whereas at high latitudes phytoplankton show no response to changes in nutrient supply.

The zooplankton grazing rate is given by

$$
G(P)=\frac{g \varepsilon P^{2}}{g+\varepsilon P^{2}}
$$

where $g$ is a maximum grazing rate for when $P$ becomes large, and $\varepsilon$ is the slope of $G(P)$ for small $P$.

The terms on the right-hand side of Eqs. (2)-(5) are parameterizations of different biological processes with different levels of accuracy. The photosynthetic terms are considered to be well parameterized relative to the recycling parameterizations (Evans and Garçon, 1997). In particular, for this simple model, we know very little of how well we model the time-evolution of zooplankton, the sinking rate of detritus and the remineraliza- 
tion of detritus. We will eventually test the accuracy of these parameterizations through data assimilation. As a first approach, the most poorly known biological model parameters are selected as control parameters for the assimilation:

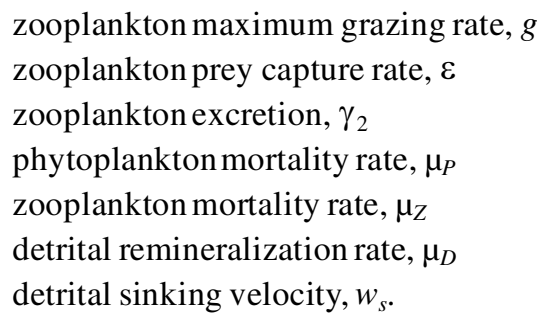

These parameters all affect the rate at which nitrogen flows out of $P$, is recycled through $Z$ and $D$, and then either sinks or goes into $N$. For the modeling work performed so far (Oschlies and Garçon, 1998, 1999) and for this study, the biological model parameters are constant in time and space.

\section{Fitting the model to simulated North Atlantic Ocean color data}

To assimilate an eddy-permitting basin-scale coupled physical/biological model with a basin-scale ocean color data set, on seasonal time scales, is a formidable task. Given the large CPU time and memory demands associated with a full 4-D assimilation of this scale, and given that our first objective is to investigate the sensitivity of the different parameters to ocean color data, we choose to adopt a computationally feasible approach.

\section{a. Lagrangian water columns}

Our approach is to seed several floats in different regions of the model domain and follow their trajectories with a one-dimensional biological model that includes vertical advection and vertical diffusion. That is, the floats are seeded just below the surface in the ocean model, and the water column above which they sit is advected around by the velocity field provided by the top level of the physics. Compared to previous assimilation studies, here we implicitly include the influence of horizontal advection on the biological tracers. The magnitude of horizontal diffusion is assumed to be much smaller than advection or the source-minus-sink terms which is a reasonable assumption for an eddy-permitting model. In fact, Fasham et al. (1990) showed that even in their relatively coarse-resolution model, contributions from horizontal diffusion were smaller than contributions from horizontal advection or biological source-minus-sink terms. We assume that the initial water column follows the float, that is, there is very little vertical shear in the top levels of the ocean model (this assumption will be discussed later in this section). The 1-D model is thus the biological tracer equations (1)-(5) as functions of $z$ and $t$, with the horizontal advection and horizontal diffusion terms removed.

As a first step, the full 3-D coupled model is run over a six-month period, from autumn 


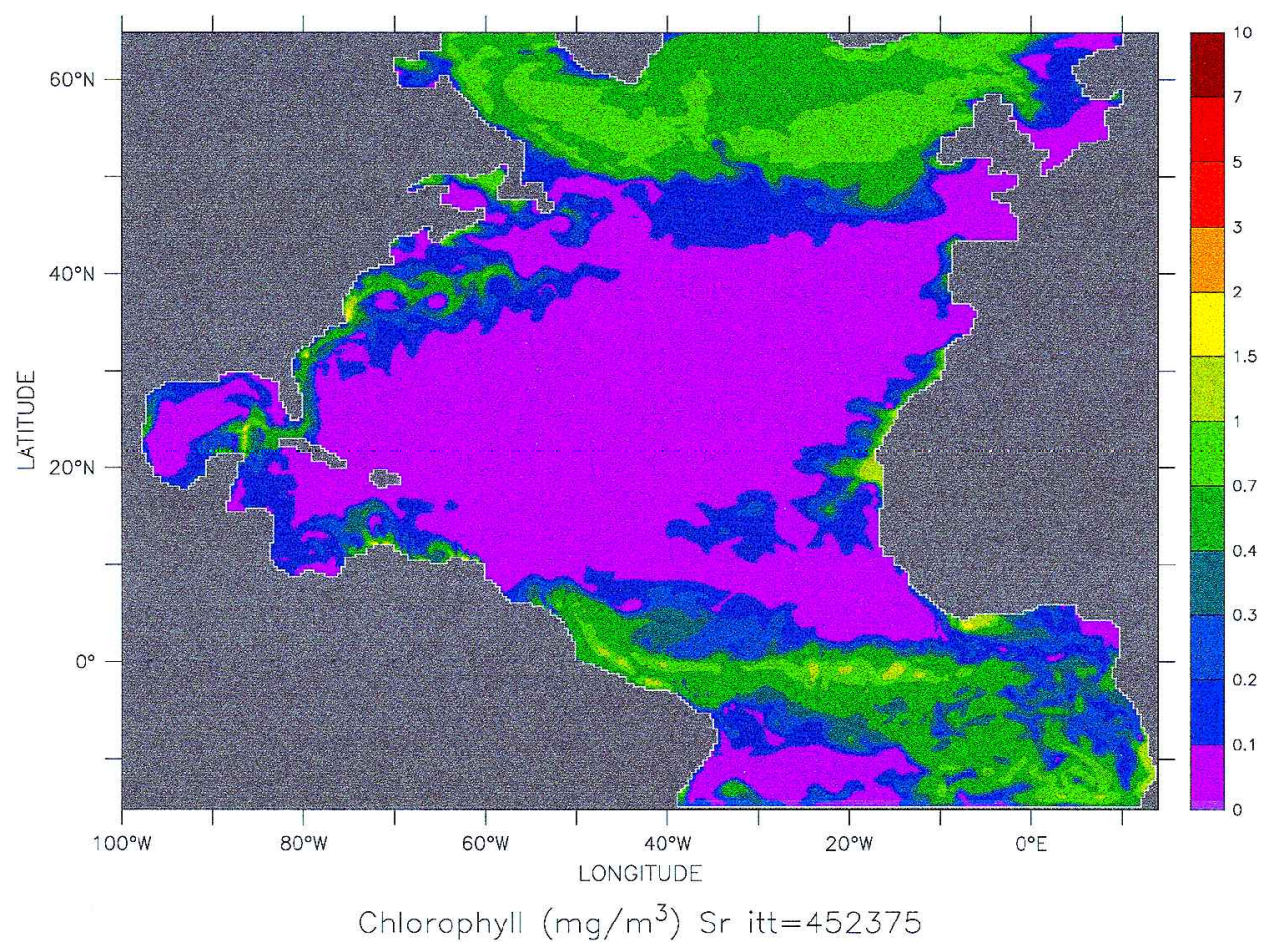

Figure 1. Modeled surface chlorophyll $\left(\mathrm{mg} \mathrm{Chl} / \mathrm{m}^{3}\right)$, on October 2, at the start of the six-month model run.

through early spring (Oct 2-Apr 2), with climatological forcing. The field of surface chlorophyll produced by the model at the start of the six-month period is shown in Figure 1. There is characteristically low surface chlorophyll concentrations over most of the North Atlantic during late autumn. Figure 2 shows the modeled field of surface chlorophyll on April 2, at the height of the spring bloom of phytoplankton. Notable features are the zones of intense chlorophyll concentrations in upwelling regions (equator, African coast). For the North Atlantic, this six-month period captures the depth of winter and the spring bloom.

From this model run the following quantities are interpolated onto the locations of the floats and stored in order to run the 1-D model following each float: initial profiles of $N, P$, $Z$ and $D$, and time-dependent profiles of temperature, vertical diffusion coefficient and vertical velocity. The latter three are needed as functions of depth and time for the calculation of the photosynthetic growth rate, the vertical advection and the vertical diffusion. Thus the mixed-layer properties, and hence the nutrient supplies, vary along the float trajectory according to the full 3-D model. However the initial inventories of $N, P, Z$ and $D$ are assumed to follow the float.

Figure 3 shows the trajectories of ten floats over the six-month period. Their spatial extent may be described using the terminology of Platt et al. (1995): floats 1-4 are situated 


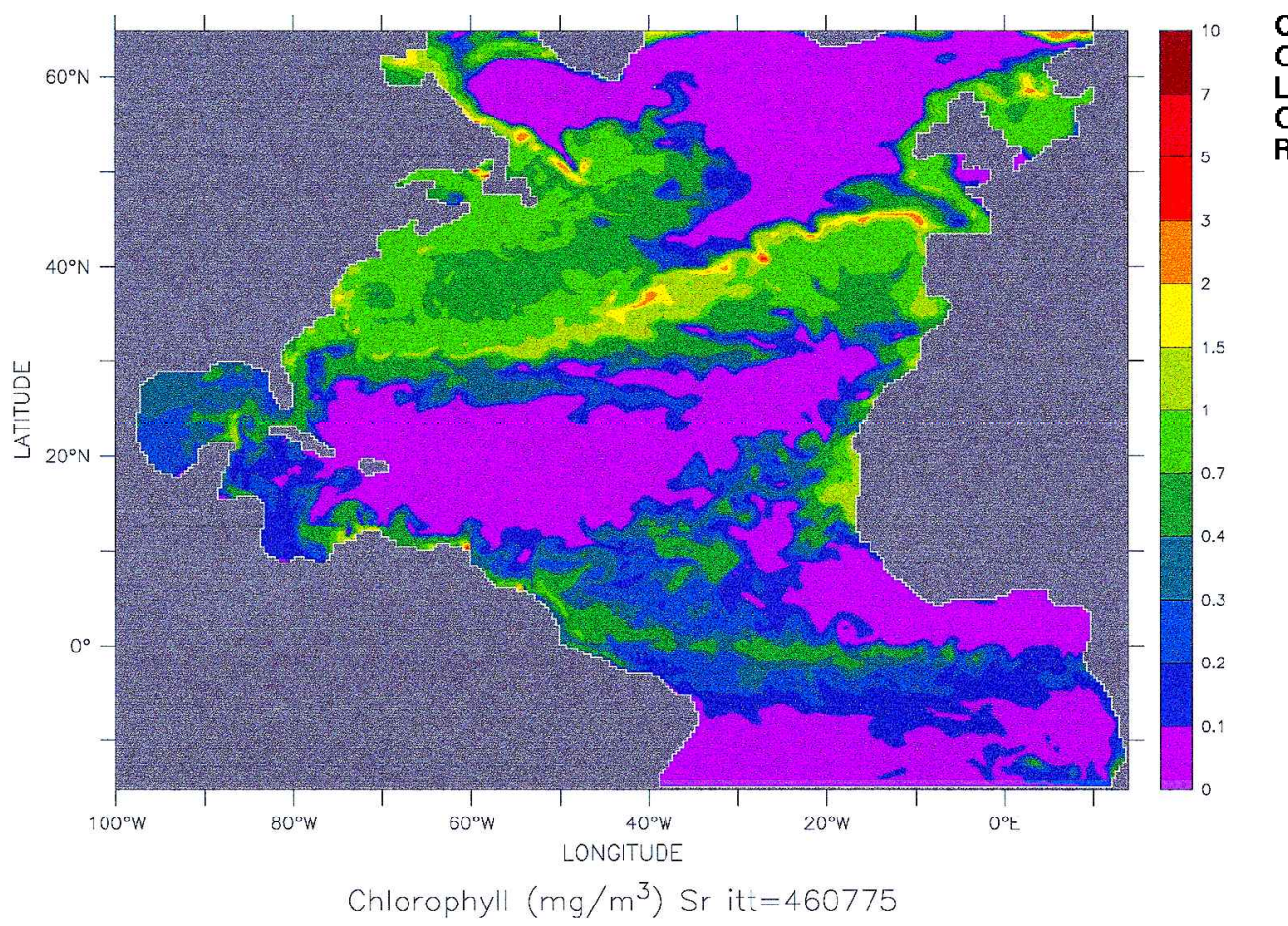

Figure 2. Modeled surface chlorophyll $\left(\mathrm{mg} \mathrm{Chl} / \mathrm{m}^{3}\right)$, on Apr 2, during the spring bloom of phytoplankton.

in the trade wind domain, floats 5-8 are situated in the westerly wind domain and floats 9 and 10 are situated in the polar domain, although there is clearly some overlap between domains.

To compare the degree of similarity between the 3-D and the 1-D model, Figures 4, 5 and 6 show time series of the biological tracers for floats 2,7 and 9, respectively. The degree of similarity between the temporal evolution of the tracer concentrations in both models appears to be best at higher latitudes. The discrepancy between the 1-D model and the 3-D model is of the same order everywhere, but is more marked at low latitudes where all of $N$, $P, Z$ and $D$ are of much lower amplitudes.

In looking for reasons for the discrepancy between the models, the main reason would be vertical shear in the top levels of the 3-D model. It was found that for at least the top three levels of the 3-D model there is very little vertical shear in both the horizontal velocity fields and in the biological tracer fields. The former was found by seeding a float between the second and third levels directly beneath float 7 , the latter was found by inspecting the water column in the 3-D model at various float positions. The discrepancy between the 3-D and 1-D model arises due to vertical shear at greater depths. As there is significant vertical shear in the horizontal velocity field at the base of both the Ekman layer and the mixed 


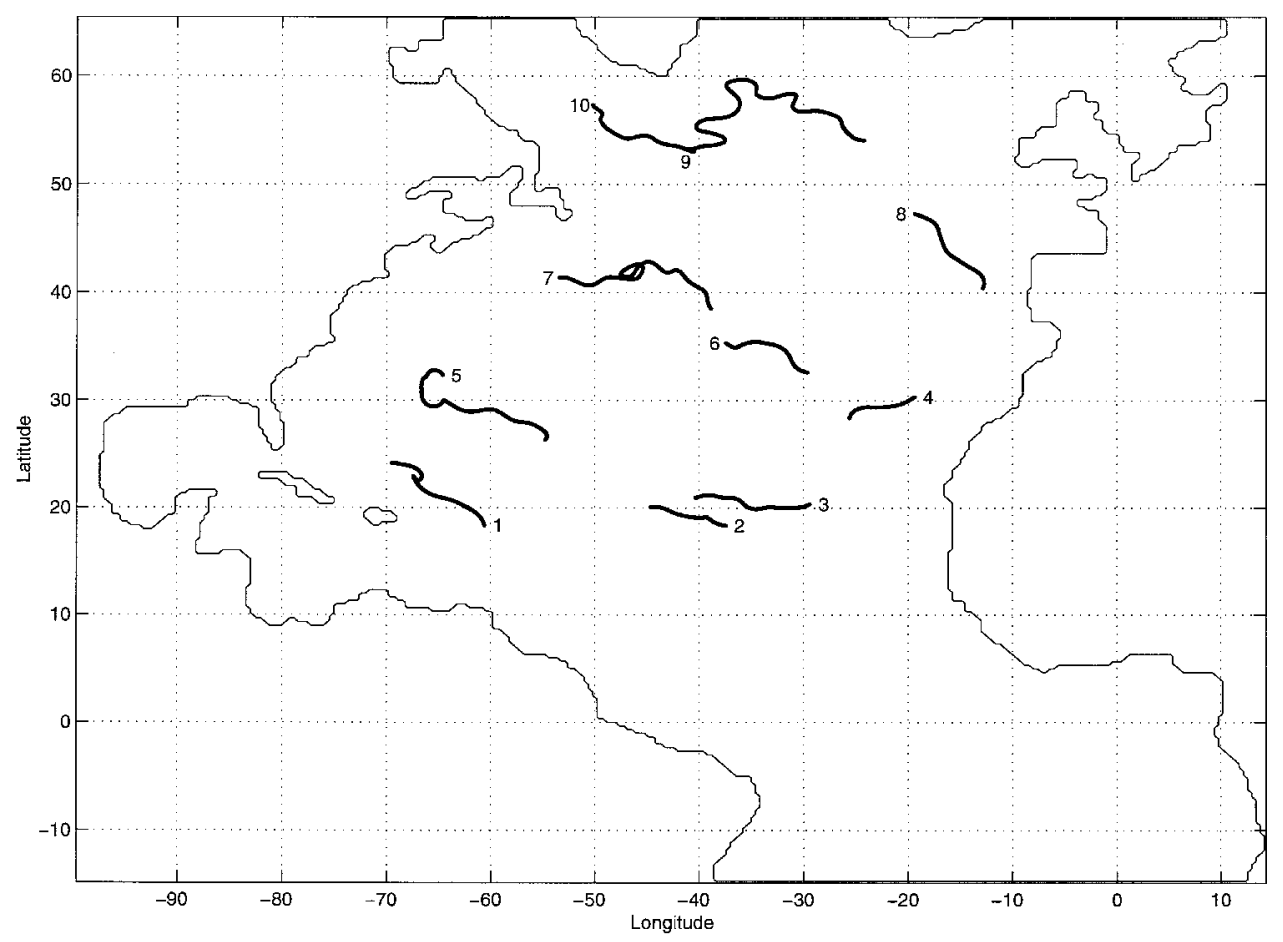

Figure 3. The domain of the North Atlantic Ocean model, showing trajectories of ten floats over a six-month period from October 2 to April 2. The float number is situated at the start of the trajectory.

layer, the water column that started with the surface float may lose its assumed vertical coherence. This is noticeable around the subtropical gyre where the phytoplankton growth rate is nutrient-limited and there is a deep chlorophyll maximum and the nitrate fields go to relatively higher values at depth. The top levels of both the 3-D and 1-D models are sensitive to variations in $N$ and $P$ at deep levels. At higher latitudes, where the phytoplankton growth rate is light-limited and the order of magnitude of all biological tracers is high in the top levels, the 3-D and 1-D models are less sensitive to variations in $N$ and $P$ at deep levels.

General biogeographical features of the surface of the North Atlantic Ocean for the October-April period are apparent in Figures 4, 5 and 6. Following float 2 in the trade wind domain, surface nitrate levels are very low, limiting phytoplankton growth. Also there is very little vertical mixing to replenish the surface nitrate consumed by the phytoplankton. Float 7, coming out of the Gulf Stream in the westerly wind domain, has much higher surface nitrate levels. Strong, local, vertical mixing events occur as winter progresses, deepening the mixed layer and supplying nitrates to the surface phytoplankton. One sees a bloom of phytoplankton following the first mixing event around day 20 that causes strong responses in zooplankton and detritus. Further increases in surface nitrates do not affect the 

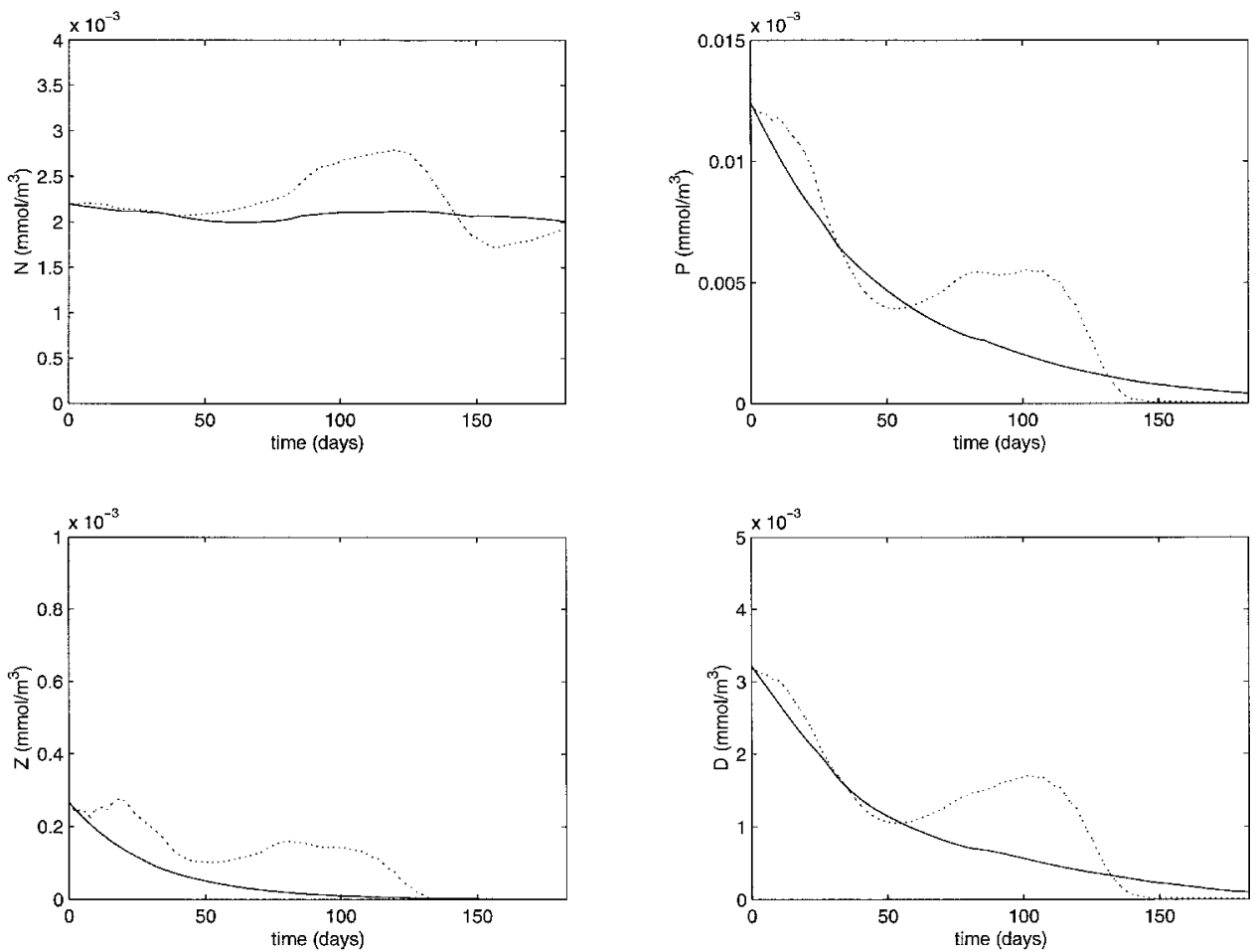

Figure 4. Time series of $N, P, Z$ and $D$ over six months (Oct 2-Apr 2) for float 02, (solid) from the surface level of the 1-D model following the float trajectory and (dotted) from the full 3-D model interpolated onto the float location.

levels of phytoplankton, zooplankton and detritus, as the phytoplankton growth rate becomes light-limited in the middle of winter. The sudden spring bloom may be seen towards the end of the period. Float 9 , in the polar domain, shows very high nitrate levels as winter mixing progresses to the point where surface nitrate levels are the same as at depth. Light-limited phytoplankton growth causes the strong decline in phytoplankton,zooplankton and detritus, and at high latitudes the spring bloom occurs later than at lower latitudes.

The plots for floats 2, 7 and 9 are representative of their biogeographical domains, and similar behavior is seen in plots for all of the other floats that were used. Despite the small discrepancy between the 1-D and 3-D models, we feel confident that our simple approach allows us to investigate the spatial and temporal sensitivity of our ecosystem dynamics to ocean color data.

\section{b. Definition of a cost function}

The essential part of fitting a model to data is the definition of a cost function, which is a scalar measure of the misfit between the model variables and the data. There are several different methods of minimizing the cost function and the choice of method depends not 

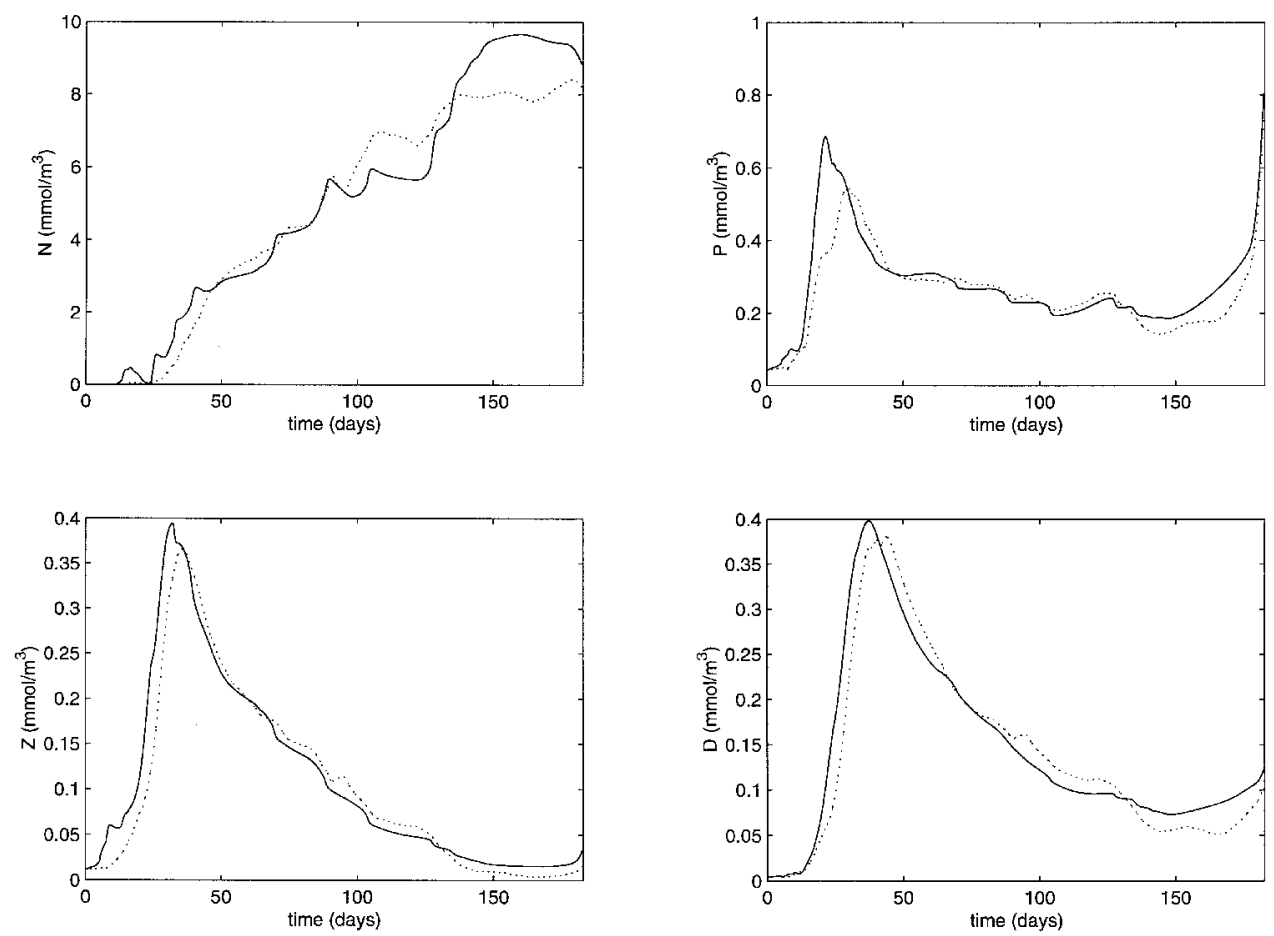

Figure 5. Same as Figure 4 for float 07.

only upon the size of the dataset, the size of the model and its degree of nonlinearity, and the availability of computing resources, but also upon what we wish to learn. As a first approach we will apply strong-constraint variational data assimilation, otherwise known as the adjoint method, in order that we can use the gradient information given by the adjoint variables to investigate the sensitivity of model parameters to ocean color data. Later we may use a weak-constraint approach, in order to also examine the role of error in Eqs. (2)-(5). With a weak-constraint approach (see Bennett, 1992) the errors in the temporal evolution of each biological variable, are also included as control parameters. For such an approach care must be taken to ensure conservation of total nitrogen.

We will now formulate the adjoint method of fitting the model equations (1)-(5) to spatially and temporally varying fields of chlorophyll $a$. The cost function consists of two parts, the first part represents the misfit between the model and the data, the second part supplies a priori knowledge of the control parameters (e.g., positiveness of grazing rates, upper physiological bounds on growth rates).

For the model/data misfit, we write a functional relationship between surface chlorophyll concentration and the state variable $P$ in the topmost layer of the 1-D model at observation times. The ratio of the surface concentration of chlorophyll $a, \operatorname{Ch}(x, y, t)$ 

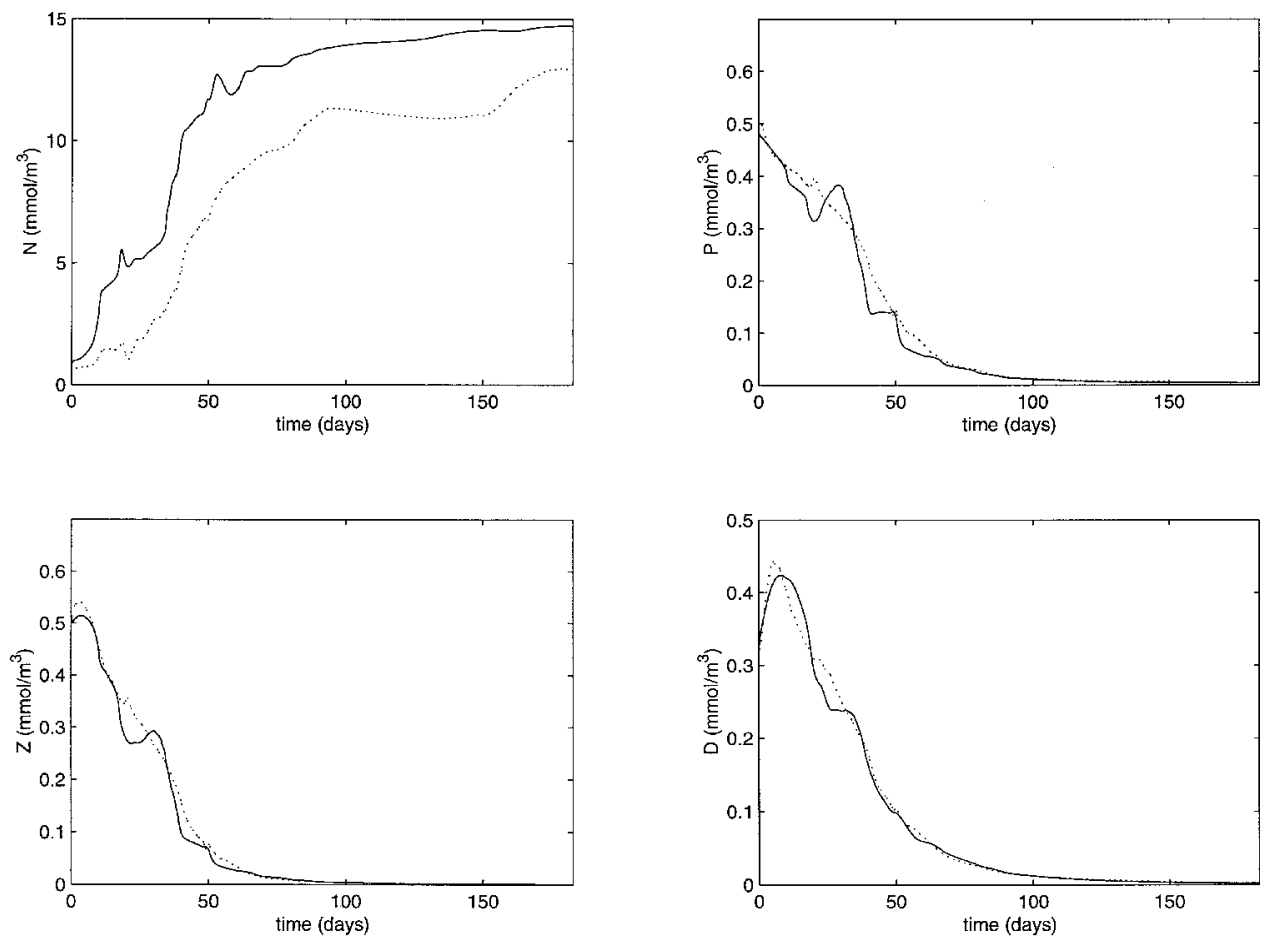

Figure 6. Same as Figure 4 for float 09.

$\left[\mathrm{mg} / \mathrm{m}^{3}\right]$, to phytoplankton, $P(x, y, z, t)\left[\mathrm{mmolN} / \mathrm{m}^{3}\right]$, is $C h: P=1.59 \mathrm{mg} / \mathrm{mmol}$, assuming a carbon/chlorophyll mass ratio of 50 and a carbon/nitrogen molar ratio of 6.625 .

The first part of the cost function is thus defined, for a float trajectory, as

$$
\mathscr{T}_{1}=\frac{1}{2 N_{f}} \int d \mathbf{x} \int d t\left[\frac{1.59 P(x, y, 0, t)-C h(x, y, t)}{\sigma_{C h}}\right]^{2} \delta\left(\mathbf{x}-\mathbf{x}_{f}\right) \delta\left(t-t_{f}\right)
$$

where: $\left(\mathbf{x}_{f}, t_{f}\right)$ are the locations and times of chlorophyll observations following a surface float trajectory, $N_{f}$ is the total number of chlorophyll observations and $\sigma_{C h}$ is the a priori observational error in the relationship between $P$ and $C h$. Here we define $\sigma_{C h}$ to be $20 \%$ of $\mathrm{Ch}$, which is a preliminary estimate of the observational error for SeaWiFS ocean color data (Greg Leptoukh, pers. comm.). $\mathscr{T}_{1}$ may be written more practically as

$$
\mathscr{T}_{1}=\frac{1}{2 N_{f}} \sum_{k=1}^{N_{f}}\left[\frac{1.59 P-C h}{\sigma_{C h}}\right]_{k}^{2}
$$

where the subscript $k$ refers to the residual calculated from $P$ and $C h$ at the $k$ th observation point along the float trajectory. Cost functions are defined for each float trajectory and then either minimized separately, or added together and minimized simultaneously. 
The second part of the cost function provides any a priori information we have about the seven model parameters that are to be used as control parameters. The parameters are assembled into a vector denoted by

$$
\phi=\left[g, \varepsilon, \gamma_{2}, \mu_{P}, \mu_{Z}, \mu_{D}, w_{s}\right] .
$$

The level of ignorance about the expected values of the parameters can vary substantially. Given a priori first-order statistics for each parameter (expected value, $\bar{\phi}_{i}$, and expected variance, $\sigma_{\phi_{i}}$, we explicitly include these in the second part of the cost function as

$$
\mathscr{T}_{2}=\frac{1}{2 \times 7} \sum_{i=1}^{7}\left[\frac{\phi_{i}-\bar{\phi}_{i}}{\sigma_{\phi_{i}}}\right]^{2} .
$$

The expected values are those listed in Table 2 (as used in Oschlies and Garçon, 1999). The expected variances are in general poorly known. Without further knowledge, we assume a $100 \%$ a priori variance for all of the parameters. A further step will be to determine $a$ posteriori error variances for the model parameters. These may be determined by calculating the Hessian of the cost function with respect to the control parameters at the minimum (Gunson and Malanotte-Rizzoli, 1996b).

Before becoming control parameters the seven model parameters undergo the following transformations: they are normalized by their a priori expected value, this is in order that parameters who are orders of magnitude different will be given the same weight during the minimization. Then their square root is taken as, by their physical nature, the model parameters cannot take on negative values. The control parameters are thus

$$
\psi_{i}=\sqrt{\phi_{i} / \bar{\phi}_{i}}
$$

An effect of this transformation is that we now assert that the probability distribution of the model parameters about their expected values is no longer a Gaussian, but a chi-squared distribution. An effect of this is to put greater weight on small values of $\phi_{i}$.

The total cost function is hence written in terms of the control vector, $\psi$, as

$$
J(\psi)=\frac{1}{2 N_{f}} \sum_{k=1}^{N_{f}}\left[\frac{1.59 P(\psi)-C h}{\sigma_{C h}}\right]_{k}^{2}+\frac{1}{2 \times 7} \sum_{i=1}^{7}\left[\frac{\psi_{i}-1}{\sigma_{\phi_{i}} / \bar{\phi}_{i}}\right]^{2}
$$

where $\psi$ is the vector of control parameters. On successful minimization, this form of the cost function will have a value of order unity, indicating that the model/data misfit is of the order of the observational error, and that the values of the estimated control parameters are consistent with their a priori statistics.

\section{c. The adjoint method}

Given some first guess for the control parameters, we wish to calculate the gradient of the cost function with respect to the control parameters. If the cost function was a quadratic 
form in terms of $\psi$, we could readily find the global minimum of $\mathscr{T}$. However, given that the functional $\mathbf{p}(\psi)$ is nonlinear, $\mathscr{T}$ approximates a quadratic form only within some neighborhood of the first guess for the control parameters and we need to linearize the relationship between $\mathscr{T}$ and $\psi$ in order to calculate a local gradient.

The starting point is to regard the first-order Taylor expansion of the cost function about the control parameters,

$$
\delta \mathscr{T}=\left.\frac{\partial \mathscr{T}}{\partial \psi}\right|_{\psi_{0}} \delta \psi
$$

which uses the gradient to relate a perturbation in the control parameters to a perturbation in the cost function. Here $\psi_{0}$ is a first guess for the control parameters. Note that $\psi$ does not include the initial conditions for $N, P, Z$ or $D$.

Now the calculation of the cost function, from the control parameters, may be treated as a sequence of $K$ operations, i.e.,

$$
\mathscr{T}=F^{K}\left(F^{K-1}\left(\ldots\left(F^{1}(\psi)\right) \ldots\right)\right)
$$

Defining the set of variables at step $k$, that both depend on the control parameters and influence the cost function, as $\zeta^{k}$, the $k$ th is written as

$$
\zeta^{k}=F^{k}\left(\zeta^{k-1}\right)
$$

hence $\zeta^{0}=\psi$ and $\zeta^{K}=\mathscr{T}$. For our application, this sequence of operations includes the time-integration of the 1-D model equations from the initial to the final time where the control parameters $\psi$ enter at every time step. The elements of $\zeta^{k}$ are the depth profiles of $N$, $P, Z$ and $D$ at some intermediate time step, and $F^{k}$ represents all of the physical and biological dynamics that determine $\zeta^{k}$ from the previous time step.

By representing the relationship between the cost function and the control parameters as a sequence of operations, the chain rule of differentiation allows Eq. (11) to be written as

$$
\delta \mathscr{T}=\left.\left.\left.\left.\frac{\partial \mathscr{T}}{\partial \zeta^{K-1}}\right|_{\zeta_{0}^{K-1}} \frac{\partial F^{K-1}}{\partial \zeta^{K-2}}\right|_{\zeta_{0}^{K-2}} \ldots \frac{\partial F^{2}}{\partial \zeta^{1}}\right|_{\zeta_{0}^{1}} \frac{\partial F^{1}}{\partial \zeta^{0}}\right|_{\psi_{0}} \delta \psi
$$

where the subscript 0 refers to the time-dependent values of $\zeta$ arising from the $\psi_{0}$.

Thus at any intermediate time step $k$, the perturbation in $\zeta^{k}$ due to the perturbation in $\zeta^{k-1}$ is given by

$$
\delta \zeta^{k}=\left.\frac{\partial F^{k}}{\partial \zeta^{k-1}}\right|_{\zeta_{0}^{k-1}} \delta \zeta^{k-1}
$$

The elements of the Jacobian transformation matrix $\left(\partial F^{k} / \delta \zeta^{k-1}\right) / \zeta_{0}^{k-1}$, that map a perturbation in the depth profiles of $N, P, Z$ and $D$ from one time step to the next, are just the 
linearized forms of the discrete model equations; i.e., the operations contained in $F^{k}\left(\zeta^{k-1}\right)$ linearized about $\zeta_{0}^{k-1}$. Eq. (13) are called the tangent-linear model equations.

The adjoint vector, $\alpha \zeta^{k}$, is now introduced corresponding to each of the $\zeta^{k}$. Each $\alpha \zeta^{k}$ has the same dimension as its corresponding $\zeta^{k}$, and is defined by

$$
\alpha \zeta^{k} \equiv\left(\left.\frac{\partial \mathscr{T}}{\partial \zeta^{k}}\right|_{\zeta_{0}^{k}}\right)^{*}
$$

where the asterisk denotes the transpose. Combining Eqs. (12), (13) and (14) we get the following scalar product

$$
\delta \mathscr{T}=\left(\alpha \zeta^{k}\right) * \delta \zeta^{k}
$$

Recall that we are deriving a means of calculating the gradient of the cost function with respect to the control parameters. To this end, note that from the definition of the adjoint vector $(14) \alpha \zeta^{K}=1$ and also that $\alpha \zeta^{0}=\left([\partial \mathscr{T} / \partial \psi \mid \psi] / \psi_{0}\right)^{*}$, which is the gradient that we seek.

As Eq. (15) holds for any $k$, we can write

$$
\begin{aligned}
\left(\alpha \zeta^{k-1}\right) * \delta \zeta^{k-1} & =\left(\alpha \zeta^{k}\right) * \delta \zeta^{k} \\
& =\left.\left(\alpha \zeta^{k}\right)^{*} \frac{\partial F^{k}}{\partial \zeta^{k-1}}\right|_{\zeta_{0}^{k-1}} \delta \zeta^{k-1}
\end{aligned}
$$

and hence

$$
\alpha \zeta^{k-1}=\left|\frac{\partial F^{k}}{\partial \zeta^{k-1}}\right|_{\zeta_{0}^{k-1}} \mid * \alpha \zeta^{k}
$$

which gives us a means of generating each adjoint vector (starting from $\alpha \zeta^{K}=1$ ), and hence the gradient $\left(\alpha \zeta^{0}\right)$. To do so we require the transpose of the Jacobian in Eq. (13), at each time step. The transposed tangent-linear model equations (16) are called the adjoint model equations.

Previous studies using adjoint models generally used the method of Lagrange Multipliers to construct the adjoint model equations (Gunson and Malanotte-Rizzoli, 1996a; Lawson et al., 1996). Here we construct the tangent-linear model equations by linearizing the finitedifferenced forms of Eqs. (1)-(5) at the level of the code. The linearized code is then transposed, which essentially involves reversing the order of operations within each loop of the linearized code. One may see from the tracer equation (1) that the advection and diffusion terms will cause the adjoint model to spread information from the cost function in space and time, through the agency of spatial gradients in the intermediate variables $N, P, Z$ and $D$.

Having presented the mathematical foundation for the adjoint method, a verbal description of its implementation is the following: From a first guess for the control parameters, $\psi_{0}$, the value of the cost function is calculated, $\mathscr{T}\left(\psi_{0}\right)$. The adjoint variables are then calculated in order to give the gradient of the cost function with respect to the control 
parameters. From these three quantities, a gradient-search algorithm (we use the limited memory, quasi-Newton method of Zou et al., 1993) is employed to determine a new $\psi_{0}$ that is closer to the minimum of the cost function and thus iteratively one approaches the minimum.

\section{Sensitivity experiments}

The 1-D models following the floats are now fitted to simulated observations of chlorophyll concentration using the adjoint method. Given two realizable states of the model that are significantly different, in that the rms difference between the modeled chlorophyll concentration and the observed chlorophyll concentration is greater than the observational error, one may perform twin experiments between the two states. Twin experiments test the ability of the assimilation scheme to estimate previously known control parameters, and to gauge the sensitivity of the control parameters to the data.

To generate the simulated data the model parameters to be used as control parameters are randomly perturbed away from their $a$ priori values by the order of their a priori expected errors

$$
\phi *=\bar{\phi}+\sigma[2 * \text { RAND }-1]
$$

where RAND is a randomly generated number lying between 0 and 1 .

Simulated surface chlorophyll data are then created by running the 1-D model for all of the float trajectories using the perturbed variables, $\phi^{*}$. The simulated surface chlorophyll values are then used as data in the cost function, and the adjoint method is applied to minimize the cost function.

Two twin experiments (A and B) are presented here, corresponding to two different perturbations of the control parameters which give rise to two different realizations of simulated surface chlorophyll concentration along the float trajectories. Although these experiments present a highly idealized problem, where the model and data are fully consistent with each other, they do give some indication of the range of behavior of the ecosystem model to different choices of biological model parameters.

Table 3 shows, from the top, the a priori values for the control parameters and their $a$ priori expected errors, then the perturbed values of the control parameters for experiment $\mathrm{A}$ and the estimated control parameters obtained by minimizing the cost function for each float trajectory separately and then for all floats simultaneously. The same lines for experiment B are found just below. Figures 7 and 8 show the time series of chlorophyll concentration, following each float for the two experiments, arising from the different control parameters.

For both twin experiments the minimization of the cost function with respect to the control parameters is started from the a priori values for the control parameters. The forward and adjoint models are run iteratively and values of the control parameters are sought which give lower values of the cost function. The search terminates when the norm of the gradient falls below a preset value that is determined by running the adjoint model 
Table 3. Control parameters. A priori values and experimental results.

$\begin{array}{lclccccc} & g & \varepsilon & \gamma_{2} & \mu_{P} & \mu_{Z} & \mu_{D} & w_{s} \\ \bar{\phi} & 2.00 & 1.00 & 0.0300 & 0.0300 & 0.200 & 0.0500 & 5.00 \\ \sigma & 2.00 & 1.00 & 0.0300 & 0.0300 & 0.200 & 0.0500 & 5.00 \\ \mathrm{~A} \phi^{*} & 3.63 & 0.464 & 0.0144 & 0.0150 & 0.0313 & 0.0641 & 1.91 \\ \mathrm{~A} \phi_{10} & 2.00 & 1.06 & 0.0304 & 0.0217 & 0.188 & 0.0500 & 5.00 \\ \mathrm{~A} \phi_{09} & 2.00 & 1.07 & 0.0292 & 0.0162 & 0.191 & 0.0500 & 5.00 \\ \mathrm{~A} \phi_{08} & 2.00 & 0.984 & 0.0303 & 0.0297 & 0.200 & 0.0501 & 4.99 \\ \mathrm{~A} \phi_{07} & 1.98 & 0.935 & 0.0312 & 0.0252 & 0.199 & 0.0500 & 5.00 \\ \mathrm{~A} \phi_{06} & 1.98 & 0.911 & 0.0331 & 0.0142 & 0.202 & 0.0557 & 4.53 \\ \mathrm{~A} \phi_{05} & 1.98 & 0.820 & 0.0348 & 0.0129 & 0.203 & 0.0587 & 4.31 \\ \mathrm{~A} \phi_{04} & 2.00 & 1.00 & 0.0300 & 0.0129 & 0.200 & 0.0529 & 4.74 \\ \mathrm{~A} \phi_{03} & 2.00 & 1.00 & 0.0307 & 0.0171 & 0.200 & 0.0891 & 2.24 \\ \mathrm{~A} \phi_{02} & 2.00 & 1.00 & 0.0300 & 0.0123 & 0.200 & 0.0631 & 2.97 \\ \mathrm{~A} \phi_{01} & 2.00 & 1.00 & 0.0314 & 0.0306 & 0.200 & 0.0788 & 0.202 \\ \mathrm{~A} \phi_{\text {all }} & 1.34 & 0.507 & 0.0152 & 0.0150 & 0.0367 & 0.0821 & 2.62 \\ \mathrm{~B} \phi^{*} & 3.84 & 1.45 & 0.0247 & 0.0447 & 0.107 & 0.0440 & 9.33 \\ \mathrm{~B} \phi_{10} & 1.99 & 1.46 & 0.0108 & 0.0434 & 0.161 & 0.0500 & 5.00 \\ \mathrm{~B} \phi_{09} & 2.02 & 1.57 & 0.0230 & 0.0448 & 0.138 & 0.0500 & 5.00 \\ \mathrm{~B} \phi_{08} & 2.08 & 1.71 & 0.0259 & 0.0343 & 0.160 & 0.0495 & 5.04 \\ \mathrm{~B} \phi_{07} & 2.03 & 1.59 & 0.0219 & 0.0454 & 0.175 & 0.0501 & 4.99 \\ \mathrm{~B} \phi_{06} & 2.08 & 1.22 & 0.0214 & 0.0452 & 0.178 & 0.0397 & 5.93 \\ \mathrm{~B} \phi_{05} & 2.02 & 1.00 & 0.0233 & 0.0443 & 0.198 & 0.0296 & 7.18 \\ \mathrm{~B} \phi_{04} & 2.00 & 1.00 & 0.0301 & 0.0466 & 0.200 & 0.0482 & 4.62 \\ \mathrm{~B} \phi_{03} & 2.00 & 1.00 & 0.0301 & 0.0459 & 0.200 & 0.0368 & 6.72 \\ \mathrm{~B} \phi_{02} & 2.00 & 1.00 & 0.0303 & 0.0363 & 0.200 & 3.73 \times 10 & 8.22 \\ \mathrm{~B} \phi_{01} & 2.00 & 1.00 & 0.0335 & 0.0342 & 0.200 & 7.20 \times 10 & 8.67 \\ \mathrm{~B} \phi_{\text {all }} & 2.03 & 1.61 & 0.0241 & 0.0447 & 0.142 & 0.0364 & 7.76\end{array}$

using a model/data misfit of the same size as the a priori observational error. In all of the minimizations except one, the number of iterations was between ten and twenty and the cost function was minimized to well below unity which is apparent in all of the plots in Figures 7 and 8 as all of the estimated surface chlorophyll values are well within the observational error bars.

\section{a. Experiment $A$}

The randomly chosen control parameters for this experiment that were used to create the simulated data are denoted $A \phi^{*}$ in Table 3. Compared to the a priori values, $\Phi$, the difference in the $A \phi^{*}$ values that mostly affects phytoplankton growth for all floats is that the $P$ mortality rate is smaller. This generally causes the time rate of change of chlorophyll concentration to be more positive for the $A \phi^{*}$, compared to the $\phi$, by having phytoplankton live longer. Furthermore, the $D$ remineralization rate is larger and the $D$ sinking velocity is smaller than their a priori values. In the trade-wind domain these changes cause 

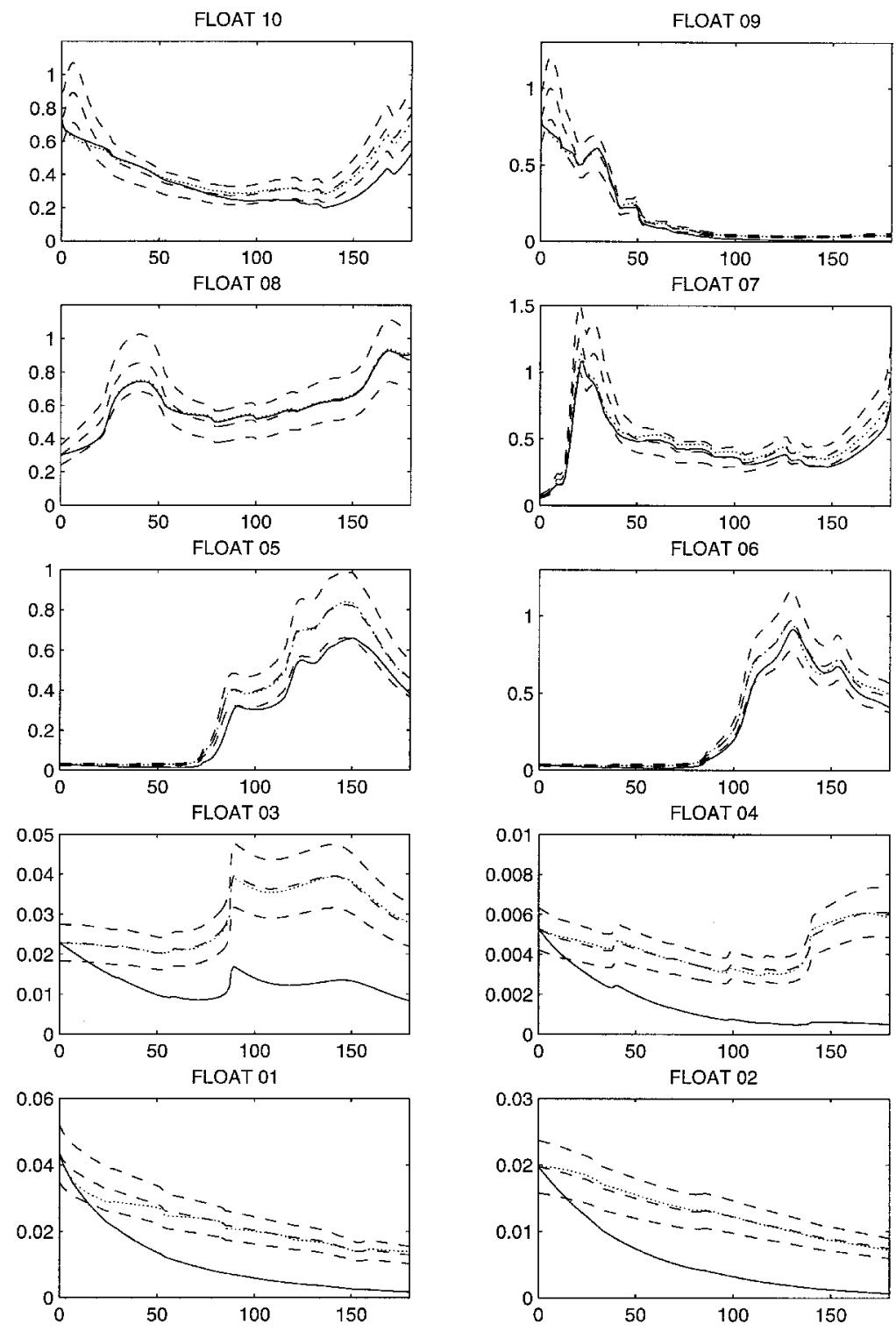

Figure 7. Simulated surface chlorophyll concentration $\left(\mathrm{mg} \mathrm{Ch} / \mathrm{m}^{3}\right)$ following each of the ten floats. Solid: from a priori values of control parameters. Dashed: from the randomly perturbed control parameters for experiment $\mathrm{A}$, the \pm twenty percent observational error level is also plotted. Dotted: from the estimated control parameters found by minimizing the cost function. 
FLOAT 10
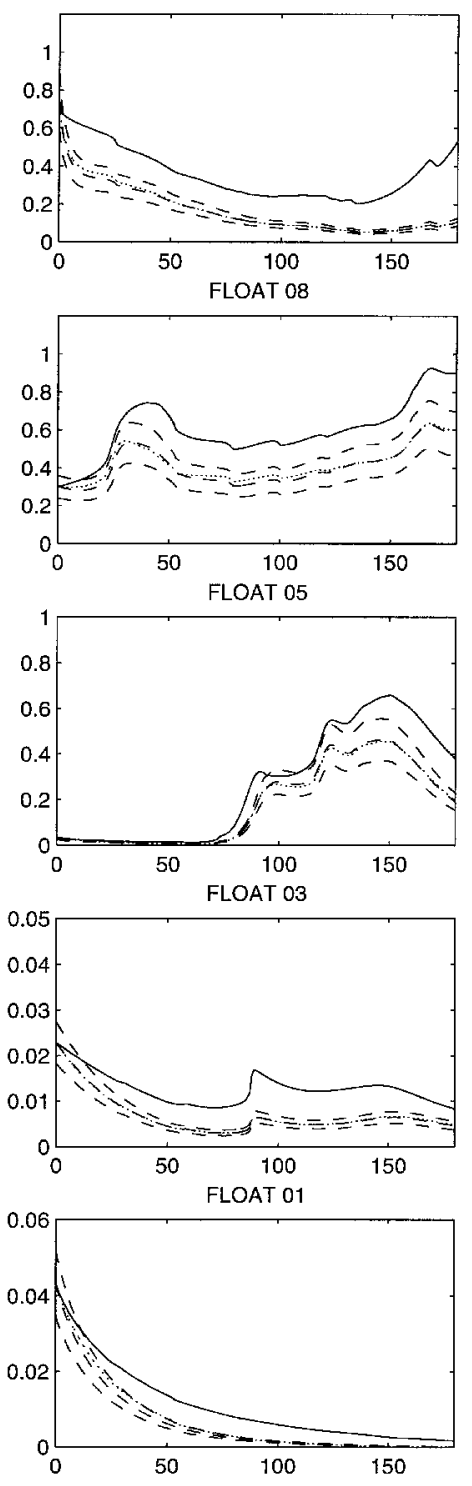

FLOAT 09
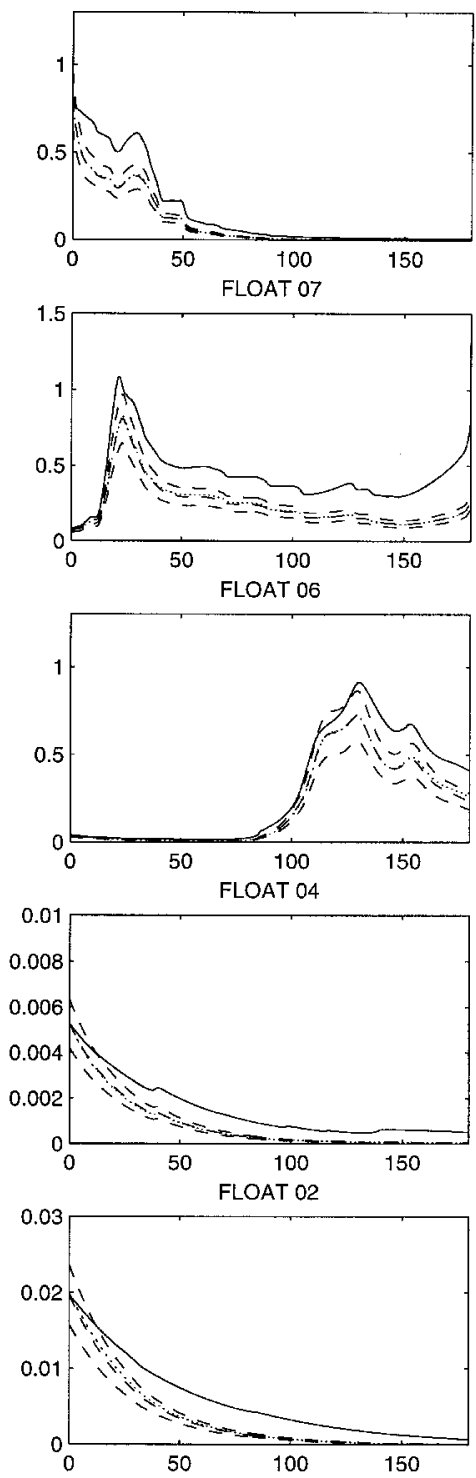

Figure 8. Same as Figure 7 but for experiment B.

an increase in chlorophyll concentrations by having more nitrogen be recycled through detritus into the nitrate pool.

Out of all of the control variables it was found that the $P$ mortality rate has the most influence on the time rate of change of chlorophyll concentration. The $Z$ parameters have much less influence except for regions where $Z$ is of the order of $P$ in which case the $Z$ 
parameters become more influential. A property of our chosen formulation of the grazing rate of zooplankton upon phytoplankton (Eq. 7) is that at low levels of $P$, as in the trade wind domain the $Z$ are relatively insensitive to changes in $P$. In the westerly wind and polar domains, at higher levels of $P$, the $Z$ are more sensitive to changes in $P$. This can be seen in Figure 7 where floats $1-5$ in the trade wind domain have simulated chlorophyll concentrations with higher net growth rates which are sustained throughout the six-month period. However the high growth rates of $P$ are soon arrested by $Z$ grazing for the floats in the westerly wind domain and polar domain (floats 6-10).

The next entries in Table $3\left(A \phi_{10}\right.$ through $\left.A \phi_{01}\right)$ are the control parameters estimated by running the assimilation along each float trajectory individually. In all cases the cost function has been minimized to order unity; i.e., the model agrees with the data to the order of the observational error. Also in each case the minimization converges to a local minimum. In some cases the minimization has changed the first-guess values toward the true values (the $A \phi^{*}$ ), but mostly the estimated values stay close to the first-guess values. In Figure 7 the curves for the estimated chlorophyll concentration are from the estimated control parameters for each float separately.

A local minimum here does not necessarily mean that the minimum is separated from the global minimum by a ridge. During the minimization, the gradient of the cost function is very low in those directions of control space that have little influence on the cost function; i.e., the null-space of the minimization. Hence the descent algorithm may stop anywhere in these flat regions where the cost function and its gradient attain sufficiently low values.

The following results may be seen from the individual float assimilations. The $P$ mortality rate is well changed away from its first-guess values toward the true value. The estimated $Z$ parameters are relatively unchanged from their first-guess values. There is a latitudinal dependence in the sensitivity of the chlorophyll concentration to the $N$ and $D$ recycling parameters. In the polar domain where the $P$ growth rate is strictly light-limited, the chlorophyll concentration is insensitive to the level of $N$. Hence the $D$ remineralization rate and sinking velocity are unchanged from their first-guess values in the polar domain (floats 9 and 10). In the westerly-wind domain the $P$ growth rate is light-limited only in the middle of winter. At the beginning and the end of the assimilation period the $P$ are sensitive to the availability of $N$, due to $P$ growth being nutrient-limited at these times. Thus the estimated $\mu_{D}$ and $w_{s}$ are slightly changed away from their first-guess values toward the true values. In the trade wind domain, where the $P$ growth rate is strictly nutrient-limited, the estimated $\mu_{D}$ and $w_{s}$ are drastically changed away from their first-guess values toward the true values for this experiment.

Finally an assimilation is performed using the simulated chlorophyll data along all float trajectories simultaneously. The estimated chlorophyll concentrations (not shown in Fig. 7) are all closer to the simulated data than the estimated chlorophyll concentrations for each float assimilated separately. The better fit for the simultaneous assimilation arises from the fact that there are more data available for the same number of unknown parameters and hence a better fit is made than for the individual assimilations. That is, using all of the floats 
the minimization of the cost function goes toward the global minimum. For the individual assimilations the cost function is minimized to a local minimum that reflects the strongest gradients of the cost function with respect to the control parameters for that biogeographical province. For the simultaneous assimilation there are contributions to the gradient from all parts of the ocean and hence one would think that the cost function goes to a minimum that is more likely to be closer to the global minimum. The better fit to each individual float for the simultaneous assimilation is due essentially to our "true" parameters having unique values over the entire basin. One of the most interesting questions, in the application of our method to actual data, will be to see to what extent this hypothesis holds in reality.

Note that the above explanation is not completely applicable. The estimated control parameters, $A \phi_{\text {all }}$, are all changed toward the true values, except for the $Z$ maximum grazing rate which ends up farther away from the true values than when it started the minimization. The nonlinearity of the ecosystem dynamics may cause some parameters to be estimated further away from their true values.

\section{b. Experiment $B$}

For this experiment, the $B \phi^{*}$ in Table 3 are the values used to create the simulated surface chlorophyll data. The main difference in the $B \phi^{*}$ is that the $P$ mortality rate is larger giving rise to simulated surface chlorophyll concentrations that have a more negative time rate of change than that produced by the $\bar{\phi}$. Also, the $D$ remineralization rate is smaller and the $D$ sinking velocity is greater, than their a priori values. The combined effect of these changes, on the recycling of nitrogen through the model ecosystem, is to kill $P$ off more quickly and then sink their corpses more quickly, recycling less through $N$. The low net rates of $P$ growth are sustained throughout the six-month period for all floats.

The estimated control parameters are shown in Table 3. Similar results as for experiment A are obtained here: the individual float minimizations converge to local minima where the model/data misfit is of the order of the observational error. The estimated chlorophyll concentrations for all floats assimilated simultaneously (not shown in Fig. 8) are all closer to the simulated data than the estimated chlorophyll concentrations for each float assimilated separately.

The $P$ mortality rate is well estimated, and the $D$ parameters show similar biogeographical dependence as for experiment A. The main difference with this experiment is that for floats with high levels of $P$, the $Z$ prey capture rate, excretion rate, and mortality rate are well changed away from their first-guess values toward the true values for this experiment. Where vertical mixing has caused phytoplankton blooms, zooplankton respond and their concentration grows to the point where changes in the $Z$ parameters have some effect on the time rate of change of chlorophyll concentration. This was not apparent in experiment $A$ as there the floats with high levels of $P$ do not have a large model/data misfit at the start of the assimilation. The minimization of the cost function in experiment $\mathrm{A}$ was dominated by the large model/data misfit for the trade wind floats which had very low levels of $Z$, and hence perturbations in the $Z$ parameters did not influence the time rate of change $P$ greatly. 
Table 4. Gradient of the cost function for the experiments listed in Table 3 at the start of the minimization.

$$
\frac{\partial \mathscr{T}}{\partial g} \quad \frac{\partial \mathscr{T}}{\partial \varepsilon} \quad \frac{\partial \mathscr{T}}{\partial \gamma_{2}} \quad \frac{\partial \mathscr{T}}{\partial \mu_{P}} \quad \frac{\partial \mathscr{T}}{\partial \mu_{Z}} \quad \frac{\partial \mathscr{T}}{\partial \mu_{D}} \quad \frac{\partial \mathscr{T}}{\partial w_{s}}
$$

$\begin{array}{llllllll}\text { A 10 } & 0.15 & 6.71 & -4.14 & 20.7 & -2.52 & -10^{-16} & -10^{-16} \\ \text { A 09 } & 0.48 & 11.9 & -3.51 & 43.3 & -5.14 & -10^{-16} & -10^{-16} \\ \text { A 08 } & 0.06 & 0.52 & -0.27 & 0.30 & 0.07 & -0.09 & 0.08 \\ \text { A 07 } & 0.29 & 5.23 & -2.51 & 8.01 & -1.45 & -0.02 & 0.02 \\ \text { A 06 } & 0.06 & 0.62 & -2.22 & 15.9 & 0.04 & -3.11 & 2.67 \\ \text { A 05 } & 0.08 & 1.41 & -2.11 & 16.8 & 0.05 & -4.13 & 3.38 \\ \text { A 04 } & 10^{-7} & 10^{-4} & -0.09 & 45.4 & 10^{-4} & -3.78 & 3.40 \\ \text { A 03 } & -10^{-6} & -10^{-3} & -0.13 & 31.3 & 10^{-3} & -9.31 & 8.97 \\ \text { A 02 } & 10^{-9} & 10^{-4} & -0.09 & 37.1 & 10^{-4} & -11.4 & 11.1 \\ \text { A 01 } & 10^{-8} & 10^{-3} & -0.29 & 34.8 & 10^{-2} & -13.6 & 14.3 \\ \text { A all } & 1.11 & 26.4 & -15.4 & 254 . & -8.95 & -45.4 & 43.8 \\ \text { B 10 } & -6.72 & -412 . & 265 . & -1330 . & 166 . & -10^{-16} & -10^{-16} \\ \text { B 09 } & -77.2 & -2210 . & 709 . & -13400 & 990 . & -10^{-16} & -10^{-16} \\ \text { B 08 } & -4.00 & -53.6 & 12.6 & -7.94 & 20.2 & 0.11 & -0.11 \\ \text { B 07 } & -4.23 & -145 . & 76.2 & -232 . & 50.1 & 0.04 & -0.04 \\ \text { B 06 } & -1.19 & -16.9 & 22.2 & -144 . & 2.74 & 27.3 & -24.0 \\ \text { B 05 } & -0.82 & -16.9 & 22.6 & -166 . & -0.511 & 32.1 & -29.5 \\ \text { B 04 } & 10^{-4} & -0.15 & 14.2 & -17000 & -0.0596 & 1340 & -1300 \\ \text { B 03 } & 10^{-5} & 0.03 & 1.49 & -440 . & -0.0158 & 123 . & -120 . \\ \text { B 02 } & 10^{-5} & 0.12 & -17.3 & -15000 & -0.107 & 4960 . & -5340 . \\ \text { B 01 } & 10^{-4} & 1.19 & -99.1 & -7610 . & -3.02 & 3340 . & -3660 . \\ \text { B all } & -94.2 & -2860 . & 1010 . & -55300 . & 1230 . & 9820 . & -10500 \\ \text { - } & & & & & & & \\ \end{array}$

\section{c. Interpreting the gradient vector}

Some insight may be gained into the results that have just been described by examining the values of the gradient of the cost function with respect to each control parameter at the start of the assimilation. For each of the assimilation experiments listed in Table 3 the values of the gradient at the start of the minimization are listed in Table 4. We look at the starting values as these correspond to the model/data misfit illustrated in Figures 7 and 8.

The parameters with the largest gradient values are those that have the greatest influence on surface chlorophyll concentration at the start of the minimization. The sign of the gradient for a particular parameter indicates in which direction the parameter should be varied in order to find a relatively lower value of the cost function. That is, a positive value of the gradient indicates that a lower value of the particular control parameter will give a lower value of the cost function. The above statement is not always true for a strongly nonlinear model.

Except for float 8 , the $P$ mortality rate has a much greater gradient than any other parameter and is thus well estimated in both experiments. The one exception is for float 8 in experiment $\mathrm{B}$, here the $Z$ parameters (excluding $g$ ) all have larger gradients than the $P$ mortality rate. This may be due to the fact that float 8 has high levels of $Z$ over the six 
months, of the order of $P$. For all of the other floats the $Z$ levels are always much less than the $P$ levels.

Overall the gradient values for experiment B are much larger than those for experiment $\mathrm{A}$, which is a consequence of the model/data misfit (and hence the value of the cost function) being much greater for experiment $\mathrm{B}$ than for experiment $\mathrm{A}$ at the start of the minimization. This is due to the simulated chlorophyll data for experiment A being much closer to the a priori chlorophyll concentration, for the floats in the westerly wind domain and the polar domain.

For floats 9 and 10 (polar domain) the gradient values for the $D$ parameters are zero, reflecting the fact that at no time of the model integration do the $D$ parameters have any influence on the surface chlorophyll due to the $P$ growth rate always being light-limited. Moving down the columns of Table 4 one can see that nutrient limitation plays more and more of a role in $P$ growth, as one moves equatorward, the gradient of the $D$ parameters becomes more and more important. The floats showing high $P$ values (floats 5-10) within the six months, show significant gradient values for the $Z$ parameters. There is thus some geographical dependence of the sensitivity of the parameters to surface chlorophyll data.

\section{Discussion}

The broad objective of this work has been to investigate the parameterizations of biogeochemical processes in a basin-scale eddy-permitting coupled physical/biological model of the North Atlantic Ocean, using simulated ocean color data as a constraint on the parameter values via variational data assimilation. In particular we wanted to determine which parameters may be well estimated from ocean color data, and also test the sensitivity of the parameterizations to ocean color data, with respect to different dynamical biogeochemical provinces.

A marine ecosystem model is coupled to a general circulation model which supplies realistic physical forcing fields. Time series of simulated surface chlorophyll concentration, following ten floats seeded in the model ocean, are used as data. The adjoint code of a 1-D model that follows each float is constructed and the adjoint method is implemented in order to perform twin experiments that address the objectives described above. Gradient information from the adjoint model allows the 1-D models to be fit to the data, and also allows the sensitivity of each parameter to the simulated data in different parts of the model ocean to be investigated.

The sensitivity results we have obtained illustrate the biogeographical dependence of certain parameters in the ecosystem model. Our formulation of the phytoplankton growth rate (Eq. 6) as light- versus nutrient-limited results in a latitudinal dependence of the sensitivity of nitrate recycling parameters to chlorophyll concentration. The detrital sinking and remineralization rates were found to have no influence on chlorophyll concentrations at high latitudes and great influence on chlorophyll concentrations at low latitudes.

For the time-evolution of $Z$, our formulation of the grazing rate (Eq. 7), coupled with the loss terms, produces a threshold $P$ concentration below which $Z$ concentrations al- 
ways decline. For the parameters used here, this threshold is about $0.2 \mathrm{mmolN} / \mathrm{m}^{3}$ or $0.3 \mathrm{mgChl} / \mathrm{m}^{3}$. Hence at low latitudes the zooplankton parameters had little influence on chlorophyll concentrations. At high latitudes, where chlorophyll concentration levels reached high values, the $Z$ parameters had a great influence on chlorophyll concentrations. A different formulation for grazing could well give $Z$ parameters that are more sensitive to low chlorophyll concentrations. Lawson et al. (1996) were able, using adjoint twin experiments, to estimate the maximum grazing rate from simulated chlorophyll and nitrate/ammonium data, using an Ivlev function to represent grazing.

We deduce from these preliminary results that, for the given functional forms of growth, mortality and grazing, we will be able to estimate the following parameters from ocean color data: nitrate and detrital recycling parameters in the trade-wind domain, zooplankton parameters at higher latitudes, and the phytoplankton mortality rate in all parts of the North Atlantic Ocean. This last parameter was also relatively well estimated by Hurtt and Armstrong (1996).

The two experiments presented in this study were run over a six-month period for reasons of computational simplicity. An immediate next step is to perform the above twin experiments on a full seasonal cycle in order to test the full range of possible chlorophyll concentrations. Alternative representations of the source-minus-sink terms should be investigated. For example, here we have used a linear phytoplankton mortality to represent the rate at which phytoplankton form detritus. In reality this may well show a quadratic dependence (Evans, 1999). The method described here could help to select formulations most appropriate for basin-scale models, which is one future work direction for the JGOFS community in its synthesis mode.

The choice of which biological model parameters to use as control parameters was somewhat arbitrary, based on which parameters were most poorly known. Important parameters affecting phytoplankton growth may be included among the control parameters. Parameters involved in the relationship between chlorophyll concentration and phytoplankton concentration will need to be considered, in particular the proportionality constant between carbon and chlorophyll concentration.

To apply the approach described to actual ocean color data, the initial conditions for $N$, $P, Z$ and $D$ need to be included among the control parameters. Here we have assimilated simulated color data at every time step of the model, i.e., thirty minutes. The SeaWiFS (level 3) data are available at daily intervals and there may be gaps of up to several days due to frequent cloud cover. The strong-constraint method we use allows the presence of temporal gaps in the data without adversely affecting the assimilation, as the temporal evolution of the biological tracers is treated as being error-free. Thus, the assimilation method described here will be able to handle long gaps in the data. It is desirable eventually to apply a weak-constraint method as we know that the source-minus-sink terms are not error-free. However, it may be difficult to include the process noise in the temporal evolution equations as control parameters. Various other methods exist that may be utilized with the model and data presented here and allow process noise, such as adaptive filtering 
(see Malanotte-Rizzoli (1996) for a review) and the method of representers (Bennett, 1992).

The assimilation strategy developed in this study has obvious limitations due to the water column coherence hypothesis. We have shown that within the subtropical gyre of the North Atlantic Ocean there might be substantial vertical shear. However, we feel that this simple approach can allow us to investigate the constraining power of ocean color data at the basin-scale on different ecosystem parameterizations. Ultimately one should proceed toward an eclectic approach, whereby satellite ocean color data are combined with data from JGOFS local studies (NABE, EUMELI, BATS, etc.) using a full 4-D assimilation scheme.

Acknowledgments. We wish to thank Isabelle Dadou for useful discussions. Constructive comments were also provided by Rob Armstrong and an anonymous reviewer. This research was made possible by support from the European Union ESCOBA program (Contract ENV4-CT95-0132). The 4-D model runs were performed on the CNES Cray-YMP.

\section{REFERENCES}

Barnier, B., L. Siefridt and P. Marchesiello. 1995. Surface thermal boundary condition for a global ocean circulation model from a three-year climatology of ECMWF analyses. J. Mar. Syst., 6, 363-380.

Bennett, A. F. 1992. Inverse Methods in Physical Oceanography, Cambridge Univ. Press, NY, 346 pp.

Bryan, F. O. and W. R. Holland. 1989. A high-resolution simulation of the wind- and thermohalinedriven circulation in the North Atlantic Ocean. Parameterization of Small-Scale Processes, Proceedings Aha Hulikoa, Hawaiian Winter Workshop, 99-115.

Evans, G. T. 1999. The role of local models and data sets in the joint global ocean flux study. Deep Sea Res., 46, 1369-1389.

Evans, G. T. and V. C. Garçon. 1997. One-dimensional models of water column biogeochemistry. JGOFS Report No. 23, Scientific Committee on Oceanic Research, 85 pp.

Evans, G. T. and J. S. Parslow. 1985. A model of annual plankton cycles. Biol. Oceanogr., 3, 328-347.

Fasham, M. J. R., H. W. Ducklow and S. M. McKelvie. 1990. A nitrogen-based model of plankton dynamics in the oceanic mixed layer. J. Mar. Res., 48, 591-639.

Fasham, M. J. R. and G. T. Evans. 1995. The use of optimization techniques to model marine ecosystem dynamics at the JGOFS station at 47N 20W. Phil. Trans. R. Soc. Lond. B, 348, 203-209.

Gaspar, P., Y. Grégoris and J.-M. Lefevre. 1990. A simple eddy kinetic energy model for simulations of the oceanic vertical mixing: Tests at Station Papa and Long-Term Upper Ocean Study site. J. Geophys. Res., 95, 16179-16193.

Gunson, J. R. and P. Malanotte-Rizzoli. 1996a. Assimilation studies of open-ocean flows, 1, Estimation of initial and boundary conditions. J. Geophys. Res., 101, 28457-28472.

— 1996b. Assimilation studies of open-ocean flows, 2, Error measures with strongly nonlinear dynamics. J. Geophys. Res., 101, 28473-28488.

Han, Y. J. 1984. A numerical world ocean circulation model. Part II: A baroclinic experiment. Dyn. Atmos. Oceans, 8, 141-172.

Hellerman, S. and M. Rosenstein. 1983. Normal monthly wind stress over the World Ocean with error estimates. J. Phys. Oceanogr., 13, 1093-1104. 
Hurtt, G. C. and R. A. Armstrong. 1996. A pelagic ecosystem model calibrated with BATS data. Deep-Sea Res., 43, 653-684.

Lawson, L. M., E. E. Hofmann and Y. H. Spitz. 1996. Time series sampling and data assimilation in a simple marine ecosystem model. Deep Sea Res., 43, 625-651.

Levitus, S. 1982. Climatological Atlas of the World Ocean. NOAA Prof. Pap. 13. U.S. Govt. Print. Office, Washington, D.C., 173 pp.

Malanotte-Rizzoli, P., ed. 1996. Modern Approaches to Data Assimilation in Ocean Modelling. Elsevier, Amsterdam, 455 pp.

Matear, R. J. 1995. Parameter optimization and analysis of ecosystem models using simulated annealing: A case study at station P. J. Mar. Res., 53, 571-607.

Oschlies, A. and V. C. Garçon. 1998. Eddy-inducedenhancement of primary production in a model of the North Atlantic Ocean. Nature, 394, 266-269.

— 1999. An eddy-permitting coupled physical-biological model of the North Atlantic. Part I: Sensitivity to advection numerics and mixed-layer physics. Global Biogeochem. Cycles, 13, 135-160.

Platt, T., S. Sathyendranath and A. Longhurst. 1995. Remote sensing of primary production: promise and fulfillement. Phil. Trans. R. Soc. Lond. B, 348, 191-202.

Prunet, P., J. F. Minster, V. Echevin and I. Dadou. 1996. Assimilation of surface data in a one-dimensional physical-biogeochemical model of the surface ocean 2. Adjusting a simple trophic model to chlorophyll, temperature, nitrate, and $\mathrm{pCO}_{2}$ data. Global Geochem. Cycles, 10, 139-158.

Sarmiento, J. L., R. D. Slater, M. J. R. Fasham, H. W. Ducklow, J. R. Toggweiler and G. T. Evans. 1993. A seasonal three-dimensional ecosystem model of nitrogen cycling in the North Atlantic euphotic zone. Global Geochem. Cycles, 7, 417-450.

Zou, X., I. M. Navon, M. Berger, K. H. Phua, T. Schlick and F. X. LeDimet. 1993. Numerical experience with limited-memory quasi-newton and truncated newton methods. SIAM J. Control Optim., 3, 582-608. 